02

\title{
THE INTER- AND TRANSDISCIPLINARY PROCESS: A FRAMEWORK
}

\author{
Olivia Bina, Josefine Fokdal, Prue Chiles, Katrin Paadam and Liis Ojamäe
}

\section{Introduction}

Enabling the City aims to capture the challenges, the potential and the attraction of urban inter- and transdisciplinary research processes, and their spirit of collaboration.

In this chapter, we summarise our journey to develop a proposed framework for urban inter- and transdisciplinary processes. The framework arises largely from three sources of reflection: our collective exploration in five specific workshops, the case studies presented in Part II and the practice stories presented in Part III. It is, itself, the result of an effort in interdisciplinary inquiry, which required the building of trust, the practice of humility and the creation of a certain feeling of a shared path. This, in turn, was made possible thanks to the gift of time and space for thinking and reflection arising from all the events we shared being part of the INTREPID network and collaborators and invited speakers over four years. These events are illustrated in the diagram on the facing page (Figure I.2.1) which shows the events and their particular outcomes over the four years. These three empirical sources of reflection helped us define, in different ways, our understanding and experience of urban inter- and transdisciplinary research processes, their challenges and enabling conditions; from both an academic and more practice orientated perspective.

\section{The Journey}

The journey begins in Lisbon. In November 2015, approximately 50 scholars and practitioners from 23 countries, all with varying interests in inter- and transdisciplinary urban research and practice, met as newly appointed members of the INTREPID network in order to launch their activities. The first meeting was complex, confusing, creative and at times difficult, as it laid the ground for mutual 
understanding around INTREPID's core concepts and terms. It also marked the beginning of a joint exploration of the challenges and enabling conditions for inter- and transdisciplinary urban research and practice. This evolved into a co-designed process, ensuring collective ownership and trust between members of this rich and diverse community, who all agreed to join around a vision exploring what can enable the academy and practice to help shape sustainable cities.

Six months later, at Delft University, we began the conversation around our work. Members were invited to present research or practice that they had been involved in, with a focus on the obstacles and enabling conditions experienced in the implementation of inter- and transdisciplinarity. The discussion that followed provided the building blocks for a matrix of perceived barriers from each case study, and for the first rough draft of a co-produced framework.

In a third meeting at the Tallinn University of Technology (TalTech) we discussed methods for enabling inter- and transdisciplinary research and practice and the matrix was further developed based on new presentations of the case studies. The aim was to explore in greater detail aspects of both barriers and enabling conditions: reflecting on key methods, language, funding, culture, time, politics, structure and institutions. This led to an agreement to work together to further develop and publish the work that was being done within the group. ${ }^{1}$ An editorial group was selected to develop a book and an open call for contributions was made among the extended network.

Since authors and editors were coming from different backgrounds and cultures in urban research and practice, we planned an exercise in trust building and mutual learning in Berlin in the autumn of 2017. This "writeshop" included peer-reviewing and trust-building exercises, An important outcome was the call to relate our discussion about inter- and transdisciplinary enabling conditions in the wider context of global policy agendas for sustainable urban development, including the Sustainable Development Goals (UNGA, 2015), thus bringing into the conversation local and global perspectives and experiences (see Chapter IV.3, this volume).

A last meeting with most group members took place at the British School at Rome (BSR). ${ }^{2}$ Here, we chose to explore the different meanings and understandings of common terms we use in our work. It became clear that the range of words and concepts in need of some common basic definition was far longer than originally envisaged. A glossary was commissioned as a key part of this publication (see Chapter I.3 this volume), and, crucially, "words" became a key part of our framework. At the meeting, it was also decided that our emphasis on research and practice needed to be reflected and honoured within the book project itself, as a prerequisite for consistency. We thus identified seven practice stories from six countries linked to members of our network, which are presented in Part III of this volume. In Rome, as a smaller group of authors and editors, rather than presenting formally we discussed informally and anecdotally our wider interests and jobs and our relationship with our projects and with writing. This process led to mutual learning, building trust and self-reflection - key ingredients for any inter- and transdisciplinary process.

Looking back at where we started in Lisbon in 2015, we also recognise the intrinsically complex nature of inter- and transdisciplinary knowledge sharing and production - not least when such processes include the additional layer of cultural and linguistic diversity (see Nikulina et al., 2019). In all our events our desire to find effective ways of sharing knowledge led to establishing a combination of novel and established ways to work together: world café sessions, preparing, cooking and eating dinner together - with strictly local and biological products - "walk-abouts" and guided visits. These allowed us to relate our intellectual practices around common concerns and learning about interesting projects in each other's cities, i.e. contexts. 


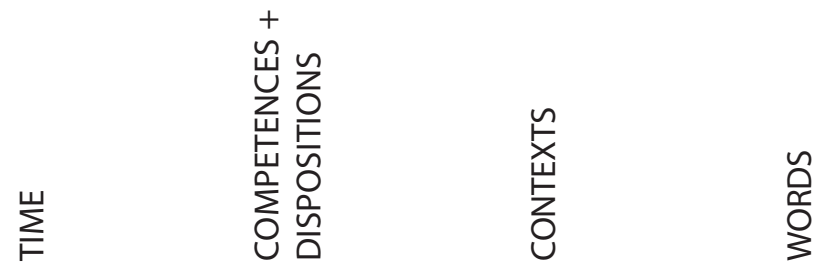

CO-DESIGN

CO-PRODUCTION

DISSEMINATION

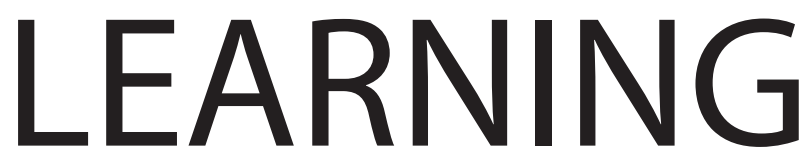

CONTINUATION

$\sqrt{u}$

FIGURE I.2.2 The elements of the framework: a journey of reflection on inter- and transdisciplinary urban processes. Source: Authors.

\section{A Framework for "Enabling the City"}

The main elements of the approach to inter- and transdisciplinary processes, developed as a result of our journey outlined above, resulted in a three-dimensional framework of what seems to matter:

- The first dimension has four phases that characterise inter- and transdisciplinary processes: codesign, co-production, dissemination and outreach, and continuation.

- The second dimension includes four enabling conditions: time, competences and dispositions, contexts, and words.

- The third dimension describes a predisposition to learning as an individual, in teams and in society: a quality that underpins and influences the workings of both the phases and the enabling conditions.

These are visualised in Figure I.2.2 and I.2.3. 
Building from experience, from a holistic perspective of knowledge production for the urban arena, and wanting to bridge boundaries between the academy and practice (including policymaking) worlds, we sought to identify those elements of inter- and transdisciplinary processes that seemed to matter the most, given our overall aim of increasing understanding and learning between disciplines and between actors and stakeholders, in academia and elsewhere.

We suggest that it is crucial to address the elements of our three-dimensional framework at different points in time - in preparation, during and after - carrying out urban research and practice. However, the list is not meant to be exhaustive, nor does it claim to provide the "right" way of undertaking inter- and transdisciplinary research or practice. Instead, it identifies the main obstacles we need to look at in actually occurring work, based on the network's cumulative experience of research and practice. For example, we do not address the issue of methods and tools here. We often discussed methods for co-designing processes and co-producing knowledge, and acknowledge that various publications on methods for integration of knowledge in inter- and transdisciplinary processes (e.g. Bergmann et al., 2012; Bammer, 2016) and on methods applied in living laboratories for co-design and co-production in transformative research processes have already been published (e.g. Defila \& Di Giulio, 2019). In addition, there are various online toolkits that are available with methods and tools for co-producing knowledge (e.g. td-net online toolkit and the Interdisciplinary Toolkit from the University of Sheffield ${ }^{3}$ ). Our discussions revealed that the real obstacles and challenges in actually implementing inter- and transdisciplinary research and practice lie in the constraints of process design, and in the capacity to turn the four dimensions of time, contexts, competences and words or language into enabling conditions.

Our proposals acknowledge and build on existing frameworks (e.g. Stauffacher et al., 2008; Jahn et al., 2012; Lang et al., 2012; Mitchell et al., 2015). Focusing on the involvement of actors in an inter- and transdisciplinary process, Stauffacher et al. (2008) state that the involvement of actors has different intensities at various times throughout the process. Other scholars have developed frameworks capturing the organisation of an inter- and transdisciplinary process (e.g. Jahn et al., 2012). Lang et al. (2012) combined the systemic understanding of the process (Jahn et al., 2012) with the need for actors' involvement (Stauffacher et al., 2008) in their framework. Yet another framework for transdisciplinary research developed by Mitchell et al. (2015) focuses on what they call "outcome spaces." These outcome spaces seek to improve a situation, to generate knowledge and to facilitate a mutual and a transformational learning experience. Building on these frameworks, we propose the three-dimensional framework "phases, learning and enablers" to help think through, plan and shape inter- and transdisciplinary processes in a more operational manner (see Figure 1.2.2).

As we go on to explain, several parts of this framework are discussed in great detail in the literature; however, through our journey we have identified specific aspects that matter most based on our experience, and that reveal the somewhat messy and blurry (not just complicated or complex) reality of undertaking inter- and transdisciplinary research and practice. The purpose is to inspire others to strive for innovative solutions to overcome the multiple barriers encountered when conducting inter- and transdisciplinary urban research and practice. In this sense, we will also want to reflect on the potential gap that needs to be filled between the lofty claims and statements in favour of inter- and transdisciplinary processes both in local and global policy documents, and the more prosaic reality on the ground. 


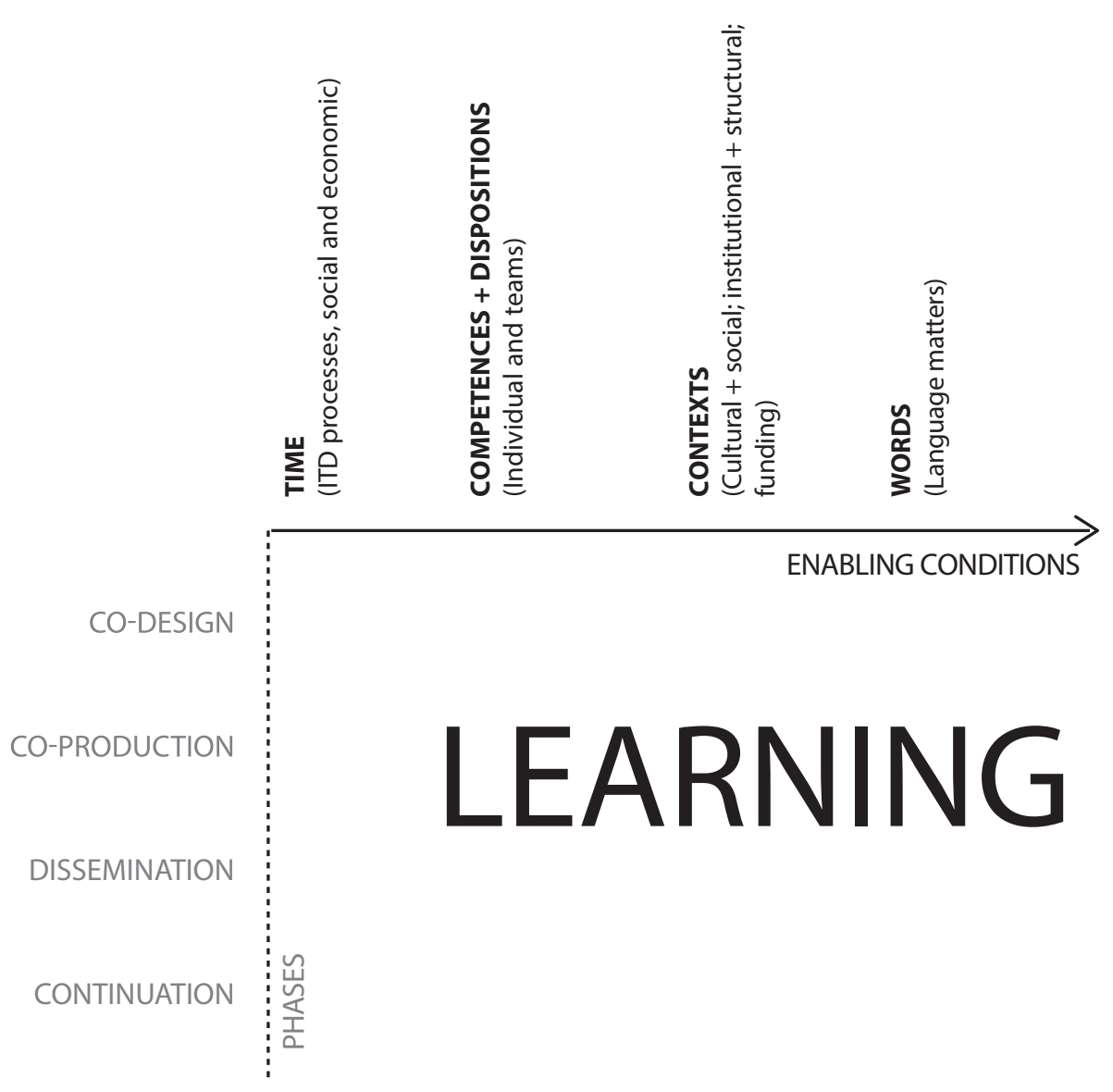

FIGURE I.2.3 Inter- or transdisciplinary process enablers: "What seems to matter." Source: Authors.

\section{A) Inter- and Transdisciplinary Phases}

The first dimension of our framework relates to four phases that characterise inter-and transdisciplinary processes: co-design, co-production, dissemination and outreach, and continuation. The first phases draw heavily on the comprehensive study "Transdisciplinary Research in Sustainability Science: Practice, Principles, and Challenges" by Lang and colleagues (2012) who identify three stages in research and related challenges: Phase 1 - Specific challenges in collaborative problem framing and team building; Phase 2 - Specific challenges in co-producing knowledge through collaborative research; and Phase 3 - Specific challenges in (re)integrating, transferring and applying the created knowledge.

As illustrated in Figure I.2.2, following discussions, it was felt that Lang and colleagues' basis needed to be enriched by one more phase: "continuation," a fourth phase that seemed to matter based on the processes actually experienced. We also interpreted their Phase 3 (dissemination and reintegration of knowledge) as a recurrent issue that is relevant throughout the whole inter- and 
transdisciplinary process and not just at the end of a process. Finally, the exploration of inter- and transdisciplinary processes, with its challenges across disciplinary and practice boundaries, required an additional aspect, which we referred to as a propensity to learning and a fruitful learning environment in which an inter- and transdisciplinary process is embedded. In Figure I.2.2 two of the three parts of the framework: A) process phases and B) the learning environment, highlight the often significant blurring of the phases as they overlap and transform each other along the life of projects.

We discuss our interpretation of each phase in turn, relating to both existing literature and some of the illustrations in Part II. As is explained in Chapter I.1, the cases in Part II were largely in progress before the framework was conceived; they are therefore ex post illustrations of elements of the four inter- and transdisciplinary phases, and of the four enabling conditions and qualities proposed here.

\section{Co-Design Phase}

The first phase is co-design (Mauser et al., 2013) or "formulating" (Polk, 2015), and it includes joint problem framing, research defining and team building (Lang et al., 2012). Based on our glossary, it is "the first phase of an inter- and transdisciplinary project ... in which the goals of the project are determined. It comprises (i) defining the central questions or problems; (ii) deciding on (the nature of) the desired answers or solutions; and (iii) identifying the knowledge and/or skills required for the answers/solutions. Besides referring to a project phase, co-design also indicates that an inclusive approach is taken, i.e. an approach in which the input of all relevant actors and the interests of all stakeholders are taken into account" (Mennes, this volume). This phase will therefore crucially benefit from the propensity to learning as a backdrop of any inter- and transdisciplinary process. In practice, this is rarely the case, especially at the start. It may be the case during the life of the project, especially if leadership (a competence discussed below) makes this a priority.

\section{Co-Production Phase}

Co-production or the co-creation of knowledge and solutions includes generating scientific integration and relevance results for policies (Mauser et al., 2013; Lang et al., 2012; Polk, 2015). In this volume, we understand co-production as the "phase of an inter- or transdisciplinary project in which answers to the central questions are generated, or solutions for the central problems are created. As with co-design, the word co-production indicates that an inclusive approach is taken, i.e. one that maintains a dialogue between all relevant actors, and, if applicable, stakeholders" (Chapter I.3).

\section{Dissemination Phase}

Dissemination (Mauser et al., 2013) or (re)integration and application of created knowledge (Lang et al., 2012) - in this volume, we talk about dissemination, which is often understood as the last phase of a project. "It is the phase in which the acquired knowledge or solution is implemented and shared" (Mennes, Chapter I.3 of this volume). However, in practice we find that the process of dissemination often overlaps with most other phases, including continuation and learning (below), thus creating a certain tension between end of project and whole process orientations. This third phase is labelled "evaluation" by Polk (2015, p. 115), thereby adding the practice-oriented dimension of evaluating the process and the impact of the results on the phases. 


\section{Continuation Phase}

Finally, we come to our suggestion to add a fourth phase - continuation - to the inter- and transdisciplinary process. Our glossary states: "Once the goals of a project have been reached and/or the funding for the project has ended, it may be decided that the project is to be continued. This continuation may consist of the writing of a new project proposal aimed at expanding or deepening the original project, or the team members deciding to prolong (and diversify) their collaboration .... In a broader sense, continuation refers to the drawing on a legacy or previous experience" (Mennes, this volume). The aspect of continuation is crucial when discussing transdisciplinarity as a timeconsuming approach against the time-limited funding schemes that most researchers are faced with. It relates to the critique of an increasingly project-oriented mode of research and practice that, while offering efficiency and output-focus investment, risks reducing knowledge production to an almost industrial plant linear process (see for example, Andersen and Kirkeby, Part II of this volume). Continuation in inter- and transdisciplinary research requires the development of more elaborate tools and trust in this approach. It could be viewed as a chain effect demanding further in-depth inquiry into the questions in focus as new questions arise, but also inspiring a wider range of self-reflexive stakeholders to be engaged in considering further opportunities for research and actual implementation of the outcomes.

Part II showcases ways of continuation within or during projects as well as after they have ended. For example, do Dietz et al. (this volume) describe a project that has achieved a certain level of sustainability by being institutionalised and thus is capable of continuing on a volunteer basis, despite the fact that the funding ran out with well-recognised actors (i.e. the university) and through dissemination of project results? Or the example by Nikšič, which describes how residents, initially misinterpreting the role of the local planning institution and hence mistrusting the initiative, demanded the invention of new approaches to continue with the project. In general, the experience of network members confirms that there is no guarantee that inter- and transdisciplinary initiatives will lead to the implementation and practical sustainability of results, since urban development depends on permanently evolving power relations being played out in daily politics. Many Part II case studies and the practice stories allude to this, despite the sometimes successful project experience (see Paadam and Ojamäe or Järg, this volume).

\section{B) Enabling Conditions That Seem to Matter}

Having introduced the four phases of inter- or transdisciplinary research and practice, and the critical dimension of learning as an individual, in teams and in society, we turn to the second part of our framework: the four enabling conditions deemed critical by network members. This is what mattered most in their experience of inter- or transdisciplinarity: time, competence and dispositions, contexts and words (Figure I.2.3). Here, too, it was agreed that a predisposition to learning, and thus a conducive environment, was essential for conditions to act as enablers.

\section{Time: The Economic and the Social}

Time matters to both inter- and transdisciplinary practices as it has a significant impact on their feasibility and effectiveness. An interesting study by Nikulina and colleagues (2019) into ways of analysing the different epistemic communities, linguistic diversities and culture in co-production agrees that "the perception of time could be a challenge" and, quoting Mikkelsen, they suggest a 


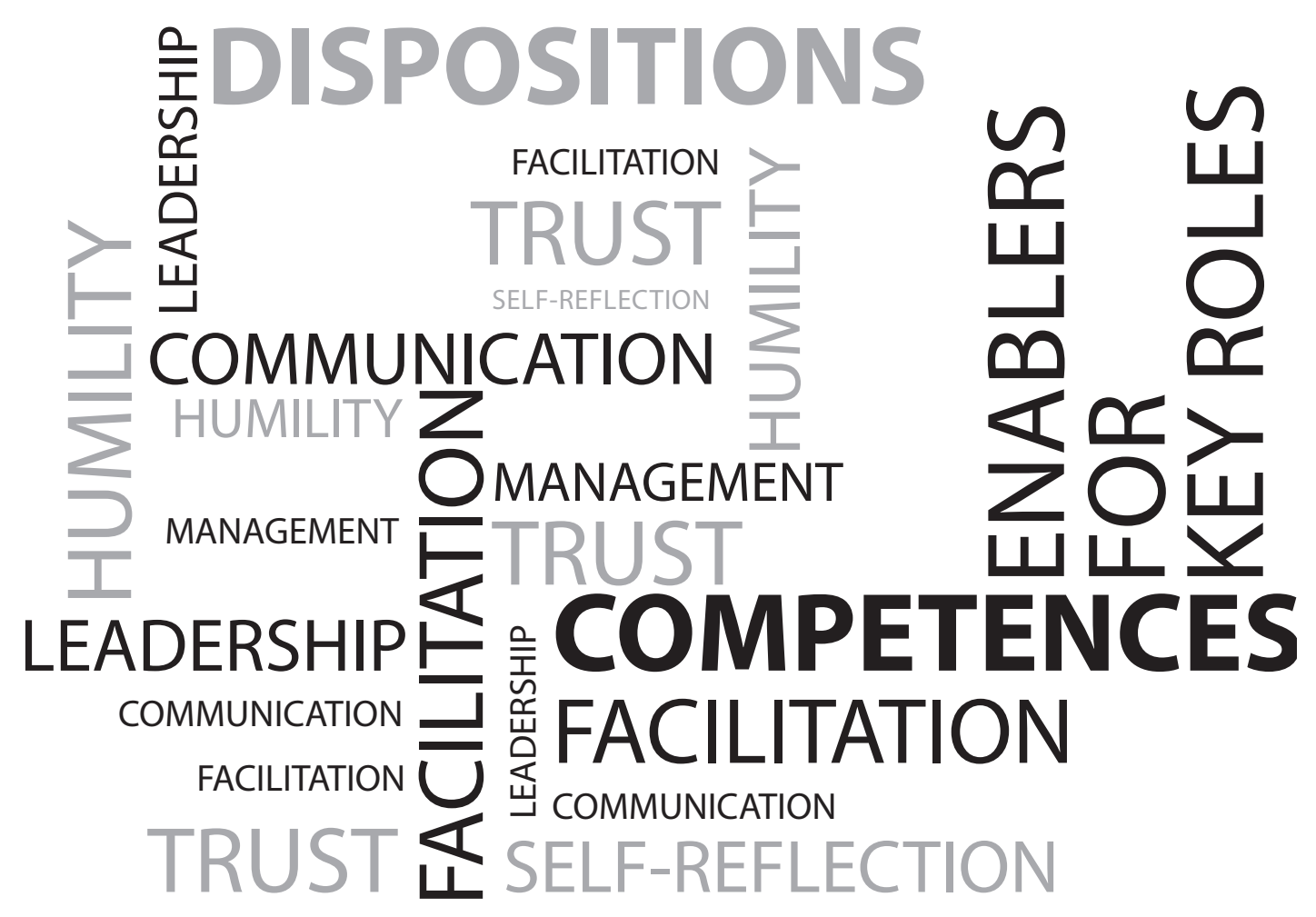

FIGURE I.2.4 Enablers: Competences (in black) Dispositions (in grey) Linked to Key Roles in ITD Processes. Source: Authors.

distinction between "economic time" and "social time." During an inter- and transdisciplinary process, time can be equated with money, and "in a Western planning model (Mikkelsen, 2005) 'economic time' plays an important role in the planning of processes" (in: Nikulina et al. 2019, p. 113). This speaks to the pervasive pursuit of efficiency, driving the "need" to produce or deliver more in less time. The intrinsic complexity and, we would argue, the often unpredictable and uncertain interand transdisciplinary processes, means that more time - not less - is likely to be needed. Indeed, an appeal for more, not less, time in European Union funding of inter- and transdisciplinary projects was one of INTREPID's key recommendations to the European Union as it planned its new research programming period (Bina et al., 2017). We discuss time as the first of our enabling conditions for inter- and transdisciplinary processes, because it is a crucial aspect in all four phases. For inter- and transdisciplinary projects linked to research funds, time is almost always too short, as the unequal weight given to economic and social time results in the former trumping the latter. Priority is increasingly given to the need to start and finish projects in (economic) time, where time spans are 
defined against mono-disciplinary parameters, virtually by default, with limited understanding of the additional demands of inter- and transdisciplinary processes in terms of social time. This theme was raised multiple times at INTREPID meetings, confirming the findings in the literature on interdisciplinary research: "A good interdisciplinary researcher will also have a high tolerance for ambiguity. This means not prematurely reducing a problem to a limited set of dimensions, but taking time to explore a range of dimensions, to test several potential boundaries to a problem (each of which may imply the involvement of different sets of relevant disciplines) until the apparently optimum boundary and set of dimensions has been identified" (Bruce et al., 2004). The dissatisfaction with lack of time is described in several cases (e.g. Dimitrova, Verdini et al., Nikšič, Paadam and Ojamäe, all this volume).

\section{Competences and Dispositions}

Our second enabling condition refers to competences and dispositions of individuals and teams (Figure I.2.4). This is a nuanced arena, where boundaries cross in terms of definitions, interpretation and practice. Based on our network's experience, our understanding of competences in inter- and transdisciplinary research processes is that they are broader than specific skills (Hartmeyer et al., 2017, in Giangrande et al., 2019, p. 3) and include two major dimensions: first, characteristics that can - at least partly - be taught (e.g. communication, leadership, facilitation and management); second, dispositions (or "dispositional thinking," e.g. syntheses of systematic, anticipatory, normative, strategic and interpersonal competences (Reid et al., 2011, in Giangrande et al., 2019, p. 5)), which cannot be taught but can be acquired through experiential learning and/or are innate characteristics of an individual (Fam et al., 2017).

In the framework, competences and dispositions are listed separately on purpose to highlight their potential distinctiveness and relevance, thus making them more clearly visible in the phase of planning for an inter- and transdisciplinary process.

a. Communication: This role includes both internal communication, i.e. among team members, and external communication, i.e. between team members and external actors and stakeholders. In different phases of the research, different challenges related to communication have been identified as the aspect of "formulating" a joint problem framing (Polk, 2015) for example, or team building (Lang et al., 2012). Enabling approaches to communication among team members and partners have been suggested by several scholars (e.g. Erichsen \& Goldenstein, 2011; Lyall \& Meagher, 2008). Less has been published on enabling approaches to communication towards external actors and non-academic partners in transdisciplinary research, for example. Here we focus on both internal and external communication, as will be illustrated in the case studies. As an enabling condition for communication, Wolf et al. (this volume) describe the easy access to the actual site of research and to information gathered by all actors. Public discussions held in different ways, as open or invited forums or workshops involving academics and different interest groups in the city, are considered informative and educating for both academia and practice. It is asserted that the educational benefits of transdisciplinary research accompanied by communication between students and stakeholders (see Dimitrova, this volume) and between students and professionals in the field (see Gromark et al., this volume) have a special value for students' academic performance and building future experts' capac-

ity. Furthermore, Verdini et al. (this volume) point to multi-fold benefits of students' participation in interactive workshops, which, by embracing diverse cultures, enables students to learn from real- 
world challenges and the local community's experiences, and the local community to learn from the participants' expertise.

b. Leadership and Management: In general, leadership and management roles include overseeing the project, making sure the timeline is respected, facilitating joint knowledge production and intervening in conflicts. These competences incorporate multiple aspects, including time availability, time management and facilitation of processes. In most of the case studies examined, it became obvious that there is an even more urgent need for someone to take leadership and to manage in inter- and transdisciplinary research than in disciplinary research (see also Polk, 2015). By management, we also mean facilitation of the process of joint knowledge production, for example, and many other aspects. The chapter by Dimitrova in this volume highlights "management of partnership" in a triad of ethics, time, funding as a serious obstacle to efficient inter- and transdisciplinary urban research. It critically reflects on the project leadership's role in the research results, and their confirmation of political decisions about the area's development. This points to all-too-common difficult negotiations of boundaries, as well as responsibilities, of the project leader and mediation between multiple actors with various interests.

The case studies reveal a wealth of dynamics related to different qualities and roles within interand transdisciplinary processes, which link to competences and dispositions. In particular, they reveal a concept of leadership and of the role of a leader within an inter- and transdisciplinary process that is in transition from a more classical understanding of leadership towards requesting competences as a facilitator of inter- and transdisciplinary processes. Thus, the role of a leader can be diverse and vary throughout the process of knowledge integration in an inter- and transdisciplinary project depending on the scope and the context. Based on our cases, we find that leadership often includes the skills and qualities of facilitation and change agents, as well as requiring several dispositions discussed here. The contribution by Anderson and Kirkeby in this volume is a good example of the dynamic roles throughout the process. They give examples of the importance of trust, open-mindedness and the willingness to listen as a basis for cooperation. By drawing on a metaphor of "orchestra leader," they point to the need for a project manager to be able to facilitate the cooperation.

The transition observed in practice is also discussed in the literature. Based on Wieser et al. (2014), Hoffmann et al. (2017) identify the following roles taken by leaders in inter- and transdisciplinary processes: collaborator, facilitator, scholar and advocator. Looking at the individual inter- and transdisciplinary researcher, Guimarães et al. (2019, p. 4, table 1), summarise a list of competences that have been identified as crucial characteristics of leaders taking the role as a facilitator. Among others, the role as a facilitator requires "commitment, connectedness, good communication and listening skills, flexibility, adaptability and capacity to build bridges." In addition, the capability "to promote learning amid the diversity of participants and to explore and clarify their differences so that dialogue and collaborative integration can occur" are crucial competences for facilitating co-production of knowledge within a team.

c. Facilitation: Facilitation is understood as a role and core competence for integrating knowledge in an inter- and transdisciplinary process. Further, it is seen as a means to reach the goal of co-producing knowledge among various actors with diverse interests and agendas. Indeed, there is significant overlap, in practice, between this and leadership, as well as with management. The role of a facilitator and the challenges of navigating in highly contested settings has been described in detail (e.g. Jordan et al., 2013). Some of the competences that they assign to facilitators are context aware- 
ness, complexity awareness, perspective awareness, process awareness and relationship awareness. In describing the case of Gagliato, Verdini et al. (this volume) point out the crucial aspect of facilitating a democratic governance process. This requires an openness towards community and the incorporation of local expertise. Dietz et al. (this volume) also focus on the role of "change agents" to facilitate transdisciplinary processes and to manoeuvre between the many fields of interests and hierarchies. They describe how a "fertile ground" is important as an enabling condition.

If leadership and facilitation often overlap in practice, facilitation is also found to share uncertain boundaries with the role of change agents. This is especially true when the purpose of inter- and transdisciplinary processes is transformational. A facilitator of knowledge within inter- and transdisciplinary processes aimed at promoting change towards a more sustainable future will often (also) be labelled as a change agent. The cases described by Dietz et al. and Verdini et al. (this volume) include facilitation as an important competence for catalysing transformation processes. Thus, the experience shared by the network suggests that facilitators can include individuals or groups with (at times) trained skills, and, at the same time, dispositions, that can have a large impact on the result of the process.

\section{Dispositions}

Figure I.2.4 combines the two aspects of the enabling condition: competences and dispositions. In a study conducted by Guimarães et al. (2019, p. 10) on inter- and transdisciplinary researchers and their motivation, attitudes, skills and behaviours linked to being involved in inter- and transdisciplinary discourse and processes, authors identified specific characteristics that cannot ordinarily be trained but can, at least partly, be learned through experience, such as understanding complex issues and linking different fields of knowledge. Our network finds that these dispositions play a large role, especially when it comes to facilitating processes of co-design and co-production of knowledge. Without them, a facilitator might not be able to provide the "safe space" that is needed in order to build trust and provide the environment for co-design and co-production. As mentioned earlier, we acknowledge that the two dimensions of competences and dispositions are not always easily distinguished. Based on the experience of the network, three dispositions were identified as especially critical to inter- and transdisciplinary processes: self-reflection, trust and humility. These qualities are highlighted below in relation to the framework's third overarching element of "learning."

a. Self Reflection: The ability to reflect on the process of co-production of knowledge on an individual level as well as on a group level has been identified as crucial, especially in terms of awareness of power relations and aspects of relationship building, discussed in detail by Polk (2015) and, in the broader context of social research, by May and Perry (2011). Several cases in Parts II and III touch upon aspects of self-reflection as an important skill and disposition of those involved in inter- and transdisciplinary processes, and the main lesson is that there needs to be more adequate attention and resources (including that of social time, above) devoted to it.

b. Trust: Wolf et al. (this volume), describe what in our broader discussions felt like a rather unique process of continued self-reflection combined with trust: an opportunity made possible by a project team that knew each other from previous projects. Paadam and Ojamäe (this volume) also refer to trust built on previous projects that paved the way towards experimenting with a qualitative in situ joint research between practising architects and academic sociologists, with this methodologi- 
cal experience having been critically reflected in ex post discussions within the team. Also, Dietz et al. (this volume) describe how an urban intervention in the form of a parklet created a space for reflection and meeting among various actors. This allowed for relationship building and the coproduction of knowledge about the specific neighbourhood among local authorities, residents and the research team. Nikšič (this volume) reflects on how the residents in a suburban neighbourhood were mobilised to express their interest in the area development after the first unsuccessful attempts by introducing new methods of research enabling civil engagement.

c. Humility: Finally humility is discussed mainly as a necessary trait for those working within the academic establishment, as a quality that can enhance interdisciplinary conversations, conducive to the sharing and integration of knowledge. In transdisciplinary terms, it is critical in allowing the legitimate voice of different ways of knowing to be heard, valued and counted. Chiles et al. (in Part II describe how the direction of the whole project changed when through time it became clear the community and citizen scientists approach was a more worthwhile and achievable direction to follow.

d. "Dispositional Competence": Reference to the work of Bourdieu around the notion of dispositions and habitus, sometimes used as synonyms, can help to clarify further the two dimensions of our framework. Habitus is defined as "an open system of dispositions that is constantly subjected to experiences, and therefore constantly affected by them" reinforcing or modifying them (Bourdieu and Wacquant, 1992). The word disposition can designate a way of being or a habitual state and, in particular, a predisposition, tendency, propensity or inclination to act (Bourdieu, 1999, p. 214). Dispositions and habitus are mutually conditioning categories, so that one is often defined through the other: they can be thought of as a potentiality, a desire to be, which seeks to create the conditions of its own fulfilment; they can be acquired and constituted in and through the social experience in various fields of human conduct, such as that of academia (Bourdieu, 1994; Bourdieu and Wacquant, 1992). Bourdieu's idea of dispositions also relates to notions of capacities and competence. Thus, action, despite carrying the appearance of rationality, is not always based on reason, because one is disposed to act and react to a particular situation based - either unconsciously or consciously - on one's experience and capacity: "The dispositional competence or connoisseurship is an art, like the art of thinking or living and is gained through a kind of apprenticeship involving repeated contact with the work" (Bourdieu, 1993, pp. 227-228).

We might therefore conclude that competences and dispositions are two sides, the cognitive and the experiential, of the same enabling condition. This echoes the definition of competences adopted by the United Nations Educational, Scientific and Cultural Organization (UNESCO), which combines them: "the specific attributes individuals need for action and self-organization in various complex contexts and situations. They include cognitive, affective, volitional and motivational elements; hence they are an interplay of knowledge, capacities and skills, motives and affective dispositions" (UNESCO, 2017, p. 10).

\section{Context}

Obviously, all inter- or transdisciplinary processes are grounded and situated in a specific context. Every context is broad and complex; however, we believe that it is rather important to keep contextual aspects in mind when engaging in inter- or transdisciplinary processes. First, there are different 
layers of context that are of importance: the societal and political context in which the inter- or transdisciplinary process takes place, the institutional contexts, the project context and the spatial context in which the inter- or transdisciplinary process plays out. Obviously, the latter three, institution, project and locality are all embedded in given socio-cultural, economic and political contexts. Innovativeness of a certain (inter- or transdisciplinary) process is also contextual, e.g. what can be considered a "paradigmatic change" in a certain urban planning context may be part of a more established tradition in another.

Various limitations linked to institutional capacities for transdisciplinary processes, for example, have been identified (Robinson, 2008). As several case studies in Part II illustrate, it starts from lack of shared physical space to more institutional hurdles linked to limited access to funding and a rigid administration. Wolf et al. (this volume), for example, describe the initial suspicion of university authorities towards the inter- or transdisciplinary project, as in the case of failure it would have endangered the prestige of the institution, and which required extra efforts and creativity from the research leader to apply for funding. Chiles et al. (this volume) describe how the historical and spatial contexts were crucial in starting a dialogue around new sources of energy. The practice story by Lafond et al. in Part III is an illustration of how societal and political context mattered and provided a fruitful context to develop the Spreefeld project.

\section{Words}

The fourth and final element of the framework's enabling conditions, in Figure I.2.3, is "words." In inter- or transdisciplinary processes, the importance of words and language, mainly because of the challenge of "ambiguity" linked to so many terms and concepts, cannot be overstated. In von Wehrden and colleagues (2018), we give a detailed account of how the network was confronted with significantly different interpretations of foundational words such as interdisciplinarity and transdisciplinarity. Above, we have given several illustrations of the weak and fading boundaries between, for example, key competences linked to terms such as leadership and facilitation. This, together with the additional reflections offered by Nikulina and colleagues (2019) suggests that, indeed, "words matter," especially when further challenged by epistemic diversity - almost intrinsically linked to inter- or transdisciplinary work. As a result, we have offered a shared baseline vocabulary, introduced by Mennes (I.3 in this volume), in full knowledge that the field will continue to change and adapt as words both shape, and are shaped by, use, context and cultures.

\section{C) A Propensity for Learning}

The third dimension of our proposed framework is learning, and a fruitful learning environment in which an inter- and transdisciplinary process is embedded (see Figure I.2.2). The focus here is essentially on a predisposition to learning at three scales: as an individual, in teams and in society. Having placed learning as a background to Figure I.2.2 illustrating our framework, the implication is that we consider this a fundamental quality underpinning and influencing the workings of both the phases and the enabling conditions.

The integration of knowledge across disciplines and the recognition of different types of knowledge are crucial for inter- and transdisciplinary research and practice (Mittelstrass, 2011; Andersen, 2013). Here, individual willingness to learn, combined with a certain disposition to trust, humility and self-reflection, all seem to help in promoting the necessary openness towards other disciplines 
and types of knowledge (including local knowledge). We use the expression "predisposition to learning" to summarise this.

The first phase of co-designing (see above, and Lang et al., 2012) focuses on problem definition, and here the aspect of reflexivity is a key principle that supports learning by the individual, the team and the wider social group, involving various actors aiming to overcome the normative assumptions and values applied (Lawrence, 2015). A predisposition to learning can be helped also by the creation of a conceptual model of how to exchange knowledge in the early phase of an inter- and transdisciplinary process (e.g. Heemskerk et al., 2003).

Learning through experiences and failures: while these aspects of individual, team and social learning often tend to be dealt with implicitly, the idea behind making this the third element of the framework is that it needs to become the explicit object of attention, planning and design of interand transdisciplinary processes. In Parts II and III, several cases and practice stories touch upon learning along these terms. Verdini et al., Dietz and Dimitrova (this volume) centre around pedagogical models of including inter- and transdisciplinary learning processes in urban planning education and the role of academia as facilitator of transdisciplinary processes. At the same time, social learning is an important part of the stories told by Chiles et al., Andersen and Kirkeby, and Heslop and Ged in this volume.

Finally, in Part IV, we explore further the theme of learning as central to international agendas for sustainable futures and, specifically, for urban sustainability.

\section{A Framework to Help Plan}

The three-part framework presented here is the result of insights and findings from the network's own debates and reflections over the four years of INTREPID's COST action, as well as the analysis of case studies and the experience shared in five dedicated workshops. The result is a heuristic-driven framework, explicitly intended to reflect experience of inter- or transdisciplinary processes, their obstacles and the possible enabling conditions that might help to solve them. While the main reason for developing the framework was to learn from each other as heterogenous members of a network of scholars and practitioners, we think the results summarised in Figure I.2.2 can also help in the early stages of designing an inter- or transdisciplinary process; the aim is to avoid as many obstacles and maximise the possibilities for desired outcomes to actually materialise.

Taking time to think about the three dimensions (inter- or transdisciplinary phases, enabling conditions and learning) together, as part of a complex process, rather than in isolation, will hopefully help avoid obvious pitfalls and omissions, while finding opportunities for synergies and, of course, learning. Fully aware of the many excellent sources of knowledge and guidance for inter- or transdisciplinary processes, we encourage the use of the suggested framework as a starting point for early planning. The idea is a simple invitation to allocate the often limited resources of time, competences and dispositions across (A) the four core inter- or transdisciplinary phases; (B) the crucial ongoing process of learning by individuals, teams and in society $(\mathrm{C})$ as the all-important enabling conditions.

We hope the framework can serve as a source of inspiration for researchers and practitioners to guide and conduct inter- or transdisciplinary urban research and practice and maximise learning throughout. Furthermore, given that another key interest of the wider INTREPID network is the programming and funding of such research, we see the potential for this framework to become a heuristic for funding institutions when drafting calls and evaluating research. Our framework resists the seemingly unstoppable drift towards an instrumental and reductionist pursuit of efficiency at the 
expense of the qualities of a plural, diverse and inevitably messy and complex process that builds on self-reflexivity, trust, humility and constant learning.

\section{Notes}

1 "Enabling the City" is only one of many outputs for the whole INTREPID network, this group being concerned specifically with Urban issues.

2 The British School in Rome (BSR) is an interdisciplinary research centre supporting the arts, humanities and architecture.

3 Interdisciplinary Toolkit: www.sheffield.ac.uk > idtoolkit was developed as a result of the Stocksbridge project, some aspects of which are discussed in Part II.

\section{References}

Andersen, H.T. (2013). Working Group 1: Characterisation of existing and future urban knowledge. In Nolmark, H., Muir, T., Andersen, H.T., Troeva, V., \& Schmeidler, K. Interim Report, Cost Action C20 Urban Knowledge Arena [accessed May 2017].

Bammer, G. (2016). Toolkits for transdisciplinarity: Toolkit \#6. GAIA, 25(4), 229.

Bergmann, M., Jahn, T., Knobloch, T., Krohn, W., Pohl, C. \& Schramm, E. (2012). Methoden transdisziplinärer Forschung: Ein Überblick mit Anwendungsbeispielen. Frankfurt: Campus Verlag.

Bina, O., Varanda, M., Sessa, C., Guimarães, H., \& Alexander, D. (2017). 1st INTREPID policy brief: Recommendations on integrating interdisciplinarity, the social sciences and the humanities and responsible research and innovation in EU research. COST Action TD1408. Retrieved from http://hdl.handle. net/10451/29190.

Bourdieu, P. (1993). The field of cultural production: Essays on art and literature. Cambridge: Polity Press.

Bourdieu, P. (1994). In other words: Essays towards a reflexive sociology. Cambridge: Polity Press.

Bourdieu, P. (1999/1972). Outline of the Theory of Practice. Cambridge: Cambridge University Press.

Bourdieu, P. \& Wacquant, L.J.D. (1992). An invitation to reflexive sociology. Chicago: University of Chicago Press.

Bruce, A., Lyall, C., Tait, J., \& Williams, R. (2004). Interdisciplinary integration in Europe: The case of the Fifth Framework programme. Futures, 36, 457-570.

Defila, R., \& Di Giulio, A. (2019). Transdisziplinär und transformativ forschen, Band 2. Eine Methodensammlung. Wiesbaden: VS Verlag für Sozialwissenschaften.

Erichsen, E. A., \& Goldenstein, C. (2011). Fostering Collaborative and Interdisciplinary Research in AdultEducation: Interactive Resource Guides and Tools. SAGE Open. https://doi.org/10.1177/2158244011403804

Fam, D. M., Smith, T. and Cordell, D. J. (2017). Being a transdisciplinary researcher: skills and dispositions fostering competence in transdisciplinary research and practice. In Transdisciplinary research and practice for sustainability outcomes. London: Taylor \& Francis, pp. 77-92.

Giangrande, N., White, R.M., East, M., Jackson, R., Clarke, T., Coste, M.S., \& Penha-Lopes, G. A. (2019). Competency Framework to Assess and Activate Education for Sustainable Development: Addressing the UN Sustainable Development Goals 4.7 Challenge. Sustainability, 11, 2832.

Guimarães, M. H., Pohl, C., Bina, O. and Varanda, M. (2019). Who is doing inter- and transdisciplinary research, and why? An empirical study of motivations, attitudes, skills, and behaviours. Futures, 112, 1-15, https://doi.org/10.1016/j.futures.2019.102441.

Heemskerk, M., Wilson, K. and Pavao-Zuckerman, M. (2003). Conceptual Models as Tools for Communication Across Disciplines. Conservation Ecology 7 (3).

Hoffmann, S., Pohl, C. \& Hering, J. G. (2017). Methods and procedures of transdisciplinary knowledge integration: empirical insights from four thematic synthesis processes. Ecology and Society 22(1):27. https://doi.org/10.5751/ES-08955-220127. 
Jahn, T., Bergmann, M. and Keil, F. (2012). Transdisciplinarity: Between mainstreaming and marginalization. Ecological Economics, 79, 1-10.

Jordan, T., Andersson, P. \& Ringnér, H. (2013). The Spectrum of Responses to Complex Societal Issues: Reflections on Seven Years of Empirical Inquiry. Integral Review, 9(1), 34-70.

Lang, D., Wiek, A., Bergmann, M., Stauffacher, M., Martens, P., Moll, P., Swilling, M., \& Thomas, C. (2012). Transdisciplinary research in sustainability science: Practice, principles and challenges. Sustainability Science, 7, 25-43.

Lawrence, R.J. (2015). Advances in transdisciplinarity: Epistemologies, methodologies and processes. Futures, $65,1-9$.

Lyall, C. \& Meagher, L. (2008). A short guide to troubleshooting some common interdisciplinary research management challenges. Edinburgh: The Institute for the Study of Science Technology and Innovation.

Mauser, W., Klepper, G., Rice, M., Schmalzbauer, B.S., Hackmann, H., Leemans, R., \& Moore, H. (2013). Transdisciplinary global change research: The co-creation of knowledge for sustainability. Current Opinion in Environmental Sustainability, 5, 420-431.

May, T., \& Perry, B. (2011). Social research and reflexivity: Content, consequence and context. London: Sage.

Mitchell, C., Cordell, D., \& Fam, D. (2015). Beginning at the end: The outcome spaces framework to guide purposive transdisciplinary research. Futures, 65, 86-96.

Mittelstrass, J. (2011). On transdisciplinarity. TRAMES, 15(65/60), 329-338.

Nikulina, V., Lindal, J.L., Baumann, H., Simon, D., \& Ny, H. (2019). Lost in translation: A framework for analysing complexity of co-production settings in relation to epistemic communities, linguistic diversities and culture. Futures, 113: 102442.

Pohl, C., Rist, S., Zimmermann, A., Fry, P., Gurung, G., Schneider, F., Ifejika, S., Kiteme, B., Boillat, S., Serrano, E., Hadorn, G.H., \& Wiesmann, U. (2010). Researchers' roles in knowledge co-production: Experience from sustainability research in Kenya, Switzerland, Bolivia and Nepal. Science and Public Policy, 37, 267-281.

Polk, M. (2015). Transdisciplinary co-production: Designing and testing a transdisciplinary research framework for societal problem solving. Futures, 65, 110-122.

Regeer, B. and Bunders, J. (2009). Knowledge co-creation: Interaction between science and society. A transdisciplinary approach to complex societal issues. Den Haag: Advisory Council for Spatial Planning, Nature and the Environment (RMNO).

Robinson, J. (2008). Being undisciplined: Transgression and intersections in academia and beyond. Futures, 40, 70-86.

Schauppenlehner-Kloyber, E. \& Penker, M. (2015). Managing group processes in transdisciplinary future studies: How to facilitate social learning and capacity building for self-organised action towards sustainable urban development? Futures, 65, 57-71.

Stauffacher, M., Flüeler, T., Krütli, P. \& Scholz, R.W. (2008). Analytic and dynamic approach to collaboration: a transdisciplinary case study on sustainable landscape development in a Swiss prealpine region. Systemic Practice and Action Research, 21(6), 409-422.

UNESCO (2017). Education for Sustainable Development Goals: Learning Objectives, UN Educational, Scientific and Cultural Organisation (UNESCO), Paris, http://unesdoc.unesco.org/ images/0024/002474/247444e.pdf (accessed: 22/5/17).

UNGA. (2015). Transforming our world: The 2030 Agenda for Sustainable Development. Geneva: UN General Assembly. Resolution adopted by the General Assembly on 25 September 2015.

von Wehrden, H., Guimarães, M. H., Bina, O., Varanda, M., Lang, D.J., John, B., Gralla, F., Alexander, D., Raines, D., White, A., \& Lawrence, R.J. (2019). Interdisciplinary and transdisciplinary research: Finding the common ground of multi-faceted concepts. Sustainability Science, 14(3), 875-888.

Wiek, A. (2007). Challenges of transdisciplinary research as interactive knowledge generation. GAIA, 16, $52-57$. 
Wieser, B., Brechelmacher, A. and Schendl, G. (2014). Identitäten und Rollen in inter- und transdisziplinärer Forschung und Lehre finden. In G. Dressel, W. Berger, K. Heimerl, and V. Winiwarter (eds.). Interdisziplinär und transdisziplinär forschen: praktiken und methoden. Bielefeld: transcript Verlag, 151-166. http://dx.doi.org/10.14361/transcript.9783839424841.151

Zierhofer, W. \& Burger, P. (2007). Transdisziplinäre Forschung - ein eigenständiger Modus der Wissensproduktion? GAIA, 16, 29-34. 